\title{
INFLUENCIA DE LAS EXPERIENCIAS VIVENCIADAS POR EL ALUMNADO EN EL DESEMPEÑO DE FUTURAS COMPETENCIAS DOCENTES
}

\author{
David Hortigüela Alcalá \\ Universidad de Burgos \\ Ángel Pérez Pueyo \\ Universidad de León \\ Javier Fernández Río \\ Universidad de Oviedo
}

\begin{abstract}
RESUMEN: El presente estudio, realizado en cuatro asignaturas de la Facultad de Educación de la Universidad de Burgos a lo largo del curso 20132014, analiza la percepción del alumnado sobre la transferencia que tiene el aprendizaje obtenido en la adquisición de competencias profesionales futuras, en este caso la docencia. Se realiza un pretest-postest, comprobando en qué medida su percepción hacia la transferencia del aprendizaje varía una vez han finalizado las asignaturas. Se emplea una metodología de estudio mixta, realizando un análisis cuantitativo, tanto descriptivo (medias y desviación típica por factores) como inferencial (ANOVAS), y uno cualitativo, en el que se utilizan unas entrevistas a los docentes una vez han finalizado las asignaturas. Se generan dos grupos; el A en el que se han utilizado autoevaluaciones y coevaluaciones como herramienta de seguimiento del aprendizaje, y el B, en el que no han existido procesos de evaluación formativa, siendo el profesor el único que ha participado en la evaluación y la calificación. Se observa cómo en el grupo A se obtuvo un incremento significativo en el factor relativo a la transferencia de competencias docentes, tanto en relación al pretest como en comparación al grupo B. Se concluye que la inclusión de metodologías abiertas y participativas en el aula, que posibiliten que el discente forme parte de su propio proceso de enseñanza, repercuten en una percepción más positiva del alumno sobre la transferencia de su aprendizaje.
\end{abstract}

PALABRAS CLAVE: Percepción del alumno, percepción docente, transferencia de aprendizaje, competencias docentes, aprendizaje obtenido. 


\title{
INFLUENCE OF STUDENT EXPERIENCES IN DEVELOPING SKILLS AS FUTURE TEACHERS
}

\begin{abstract}
This study, conducted in four subjects in the Faculty of Education at the University of Burgos during the year 2013-2014, analyzes the student's perceptions about the transfer of learning obtained in the future vocational skills, in this this case teaching. A pretest-posttest is done, checking how theirperception towards learning transfer changes once they have completed the courses. Mixed methodology study is used, performing a both descriptive (mean and standard deviation factors) and inferential (ANOVA) and one qualitative, in which some interviews with teachers are use once they have completed the courses. Two groups are generated; A) where they have been used as a tool peer-assessment and self-assessments and monitoring of learning, and B), in which there has been not formative evaluation process. In this case the teacher has been the only one who has participated in the evaluation and qualification. In group A has been a significant increase in factor related to the transfer of teaching skills. This change occurs in pretest and in group B. It was concluded that the inclusion of open and participatory methodologies in the classroom, that enable the learner part of their own learning process, produces a more positive impact on students' perceptions of their learning transfer.
\end{abstract}

KEYWORDS: Student perception, teacher perception, transfer of learning, teaching skills, learning obtained.

Recibido: 11/04/2015

Aceptado: $27 / 07 / 2015$

Correspondencia: David Hortigüela Alcalá, Departamento de Didácticas Específicas, Facultad de Educación, Universidad de Burgos, C/Villadiego s/n, 09001 Burgos. Email: dhortiguela@ubu.es.

\section{INTRODUCCIÓN}

Actualmente, son varias las investigaciones que acreditan la importancia y relación que ha de existir entre los aprendizajes que adquiere el alumno en el ámbito universitario y la práctica profesional que tendrá que desempeñar una vez finalizados los estudios (Boyle y Petriwskyj, 2014; Jiménez, 2009; Trede y McEwen, 2015). Esta idea es fundamental, siempre que la docencia no se utilice bajo una perspectiva únicamente utilitaria y se fomente a través de la misma una reflexión profunda por parte del alumno (Hortigüela, Pérez-Pueyo y Abella, 2015a).

Parece necesario, por lo tanto, que en las guías docentes de cada una de las asignaturas que componen las titulaciones vinculadas al ámbito educativo se ga- 
rantice la consonancia entre aquello que se enseña y lo que va a poder ser aplicado en el ámbito profesional. De este modo, Rodríguez-Sánchez (2011) establece que carece totalmente de sentido que las metodologías actualmente se estructuren a partir de la enseñanza de una serie de contenidos a través de la clase magistral, ya que un futuro docente será de todo menos un mero receptor de información (Ozcan, 2013). Es por ello que la metodología que se implante en las aulas ha de favorecer la implicación del alumno en aquello que aprende, generando una gran cantidad de recursos prácticos que, evaluados a lo largo del proceso, generen una motivación hacia aquello que se aprende (Baena, Granero, Sánchez y Martínez, 2013).Esta practicidad en las actividades favorece la conexión entre lo que aprende el alumno en el aula y la transferencia real que puede existir para la aplicación de los aprendizajes en otros contextos, existiendo así una consonancia lógica y cada vez más necesaria en el marco formativo y laboral actual (Lappalainen y Rosqvist, 2015). Para ello, estrategias asociadas a sistemas de evaluación formativa como las coevaluaciones intragrupales, intergrupales o autoevaluaciones de carácter individual o grupal garantizan una mayor implicación del alumno en el aprendizaje, siempre que se regule a lo largo del proceso (Chang, Liang y Chen, 2013).

Por ello, es transcendental que exista una coordinación entre los docentes que imparten docencia en una misma titulación, no solamente en función de los contenidos impartidos, sino en el tipo de actividades que se demandan al alumno así como en el enfoque metodológico aplicado. Estudios como el de Hortigüela, Pérez-Pueyo y Abella (2015b) reflejan una gran diversidad en las percepciones del alumno en función de la metodología recibida en las asignaturas, siendo más favorable aquella que permite más su participación y que se centra en el funcionamiento real de la práctica docente. De esto deriva la necesidad de reflexionar conjuntamente sobre la práctica, delimitando instrumentos concretos de recogida de información que ofrezcan diferentes puntos de vista sobre las estrategias empleadas en la asignatura y el ajuste entre los objetivos planteados y la adquisición de los mismos (MacDonald, 2012). Así, según un estudio de González-Garzón y Laorden (2012), la asignatura mejor valorada por los alumnos en las titulaciones que forman a futuros docentes es el prácticum, debido en parte a que permite el contacto directo con los alumnos y supone una transferencia directa al rol que desempeñarán como docentes. Por ello, y aunque es evidente que no todas las asignaturas tienen un componente práctico tan manifiesto, es fundamental vincularlas a contextos reales, garantizando la aplicación de lo que se enseña, de una manera directa o indirecta, al propio aula.

Uno de los aspectos comunes que garantiza la transferencia real a la práctica docente es la enseñanza de estrategias, recursos o elaboración de materiales que puedan ser implementados en clase, ya que provocan la adquisición de un bagaje por parte del alumno que posteriormente puede ser utilizado. Romero, Orzechowski y Rahatka (2014) recomiendan la necesidad de cambiar el proceso de enseñanza, pasando de la reproducción de una guía docente estandarizada a la adecuación de los contenidos en función de la demanda, intereses y el compromiso del alumnado. Partiendo de la idea de que la principal salida profesional que tienen los graduados en infantil y en primaria es la docencia en la educación reglada, experiencias como Janssen, Westbroek y Doyle (2015) indican el elevado interés que manifiesta 
el alumnado por conocer cómo funciona el proceso de oposición, qué tipo de material es necesario preparar y a qué tipo de pruebas deberán enfrentarse, lo que conlleva a un descontento por abordarse escasamente esta temática a lo largo de la carrera. Por tanto, y a pesar de que no se trate de instrumentalizar las asignaturas hacia pruebas o procesos selectivos concretos, sería conveniente trabajar conjuntamente sobre competencias comunes o transversales como la búsqueda de información de manera rigurosa, la manera de citar bibliografía, el modo de elaborar y exponer disertaciones o la forma de desarrollar métodos de investigación aplicados al aula (Teijeiro, Rungo y Freire, 2013). Si esto se hiciera de manera secuencial y progresiva a lo largo de cada uno de los cursos, el alumno obtendría una base más sólida sobre la adquisición de estos elementos transversales. Además, existiría una mayor coherencia en el tipo de procedimientos demandados al discente, ya que en algunos casos la exigencia es demasiado dispar sobre algunas tareas o trabajos que tienen los mismos objetivos (Torrego y Ruíz, 2011).

En este sentido, las estrategias metodológicas en el ámbito universitario han de fomentar la autonomía del alumno, ya no solo en el desarrollo y gestión de las tareas, sino en el grado de implicación que quiere tener en cada una de las asignaturas y la extrapolación del aprendizaje a otros contextos (Maritz y Jooste, 2011). Para ello, las autoevaluaciones y evaluaciones entre iguales permiten al alumnado ser consciente del trabajo que lleva realizado, asumiendo además el feedback que realizan tanto los compañeros de su grupo como el resto de los grupos, algo que provoca la mejora de las tareas y un mayor seguimiento en el trabajo (Trigueros, Rivera y De la Torre, 2012). Esto es trascendental en el futuro ámbito docente es transcendental, ya que una gran cantidad de decisiones deberán de tomarse bajo el consenso de varios agentes; alumnos, compañeros, familias. Por tanto, el aula universitaria es clave para fomentar en el alumno su toma de decisiones y su capacidad de reflexión, ampliando las posibilidades de actuar con criterio en función de la situación académica y profesional actual (Van Loon, Ros y Martens, 2013).

Por lo tanto, y partiendo de esta idea, en este estudio se incide en cómo prácticas concretas de trabajo basadas en la autoevaluación y coevaluación intra e intergrupal pueden influir en la percepción del alumno sobre el concepto de transferencia al desempeño docente, arrojando nuevos datos y líneas de trabajo sobre la temática.

\section{OBjetivos}

- Comparar la percepción que tiene el alumnado, antes y después de haber cursado las asignaturas, sobre la influencia de las experiencias vivencias en la adquisición de competencias profesionales docentes.

- Analizar en qué medida la edad, las titulaciones universitarias previas y la experiencia en las autoevaluaciones y coevaluaciones que tiene el alumnado incide en su percepción sobre la relevancia y utilidad de la experiencia recibida en el futuro ámbito profesional docente.

- Analizar la percepción docente sobre la justificación de los métodos pedagógicos empleados en sus asignaturas, incidiendo en la transferencia del aprendizaje generado en el alumno. 


\section{Método}

\section{Participantes}

Son 113 alumnos los que participan en la investigación (55.7\% mujeres y 44.3\% hombres), con una media de edad de 22.31 años (DT =2.35). Todos los estudiantes han cursado una asignatura obligatoria de segundo curso del Grado en Primaria en la Facultad de Educación de Burgos. Se han generado dos grupos; el A en el que se han utilizado autoevaluaciones y coevaluaciones como herramienta de seguimiento del aprendizaje y el B, en el que no han existido procesos de evaluación formativa y ha sido el profesor el único que ha participado en la evaluación y la calificación a lo largo del proceso. El profesor que imparte el grupo A tiene una experiencia de cinco años en el ámbito universitario, mientras que el del B de 20 años. Cada docente desde que entró en la Universidad Ileva impartiendo la misma asignatura. La muestra utilizada atiende a parámetros de normalidad, obteniendo en la prueba de ShapiroWilkun valor de $p$ de .131 .

\section{Instrumentos}

\section{Cuantitativos}

Se ha empleado la escala de formación inicial del profesorado (Castejón, Santos y Palacios, 2013). Tiene un total de 26 cuestiones categorizadas de uno a cinco, donde el uno es no/nada y el cinco es mucho. Este cuestionario ha sido validado por un grupo de expertos en la materia, realizándose un proceso de revisión contrastada hasta alcanzar el consenso. Además la prueba piloto realizada en su adaptación al presente estudio confirma el significado previsto de las variables que se pretenden medir, demostrando la utilidad práctica del instrumento. Respecto a la fiabilidad del instrumento, se obtiene un alfa de Cronbach de 0.872, superior al límite inferior que según Corbetta (2007) es aceptado como fiable. El nivel de confianza aplicado es del $95 \%$. A partir de la identificación de las variables predictivas del cuestionario se definieron operacionalmente cada uno de los ítems de los que consta el cuestionario, relacionados estrechamente con los objetivos de la presente investigación.

Para el cuestionario final se realizó un Análisis Factorial de Componentes Principales, con el fin de evaluar la bondad del ajuste de los datos. Se obtienen valores adecuados para el índice KMO de 0.802 como en el test de esfericidad de Barlett $(p>$.00). Los índices obtenidos en la matriz de covarianzas, presentaron ajustes satisfactorios para el índice RMSEA (Root Mean Square Error Aproximation) $=0.069$. En este índice, los valores inferiores a 0.05 indican un buen ajuste, y valores de hasta 0.08 representan errores razonables de aproximación. En el CFI (Comparative Fit Index) y GFI (Goodness of Fit Index), se alcanza un valor de 0.89, y 0.86 indicativo de buen ajuste.

Se realiza un análisis factorial de componentes principales, mediante el paquete estadístico SPSS, que muestra dos factores de estudio:

1. Aplicabilidad de los aprendizajes (12 ítems): este factor integra cuestiones relacionadas con la percepción que tiene el alumno acerca de la practicidad de 
las tareas, la metodología utilizada por el docente, la implicación del alumno en su proceso de enseñanza y las posibilidades existentes de extrapolar los aprendizajes adquiridos a otros contextos.

2. Transferencia de competencias docentes (14 ítems): se atiende a la relación directa existente entre los aprendizajes adquiridos en la asignatura y las posibilidades de transferir los mismos a otros ámbitos vinculados con el desarrollo profesional docente.

En cuanto al porcentaje de varianza explicado para cada uno de los factores, el autovalor inicial para el primer factor corresponde a $57.314 \%$ de la varianza, mientras que el segundo al $42.686 \%$ de la misma. En el factor 1 el ítem con mayor carga factorial es el de metodología utilizada por el docente con un valor de 0.612 , lo que representa un $37,45 \%$ de su varianza total. Sin embargo, en el factor 2 el ítem con mayor carga ha sido el de reflexión consensuada del aprendizaje, con un valor de 0.589 , es decir, un $34.69 \%$ de su varianza. La fiabilidad, mediante el alfa de Cronbach, del factor 1 es de .893, mientras que la del factor 2 es de .897 .

\section{Cualitativos}

Con el fin de obtener la percepción de los dos docentes que han impartido docencia en cada uno de los grupos y contrastarla con la valoración que ha realizado el alumnado, se aplicó una entrevista semi-estructurada al finalizar la experiencia. Se quiere indagar en los pensamientos que tiene cada profesor sobre la metodología empleada y el aprendizaje generado, profundizando en su propia perspectiva interna (Trainor y Graue, 2014). Para aplicar la entrevista se partió de una estructura inicial en la que se demandaban cuestiones relacionadas directamente con los objetivos de la investigación. Estas cuestiones fueron analizadas y consensuadas tanto en función de estudios similares como a partir de la experiencia previa de los investigadores. Con el fin de que la entrevista fuera lo más fructífera posible y los docentes se sintieran cómodos a lo largo de su desarrollo, se fueron añadiendo cuestiones relacionadas en función de la respuesta de los profesores, obteniendo así una conversación guiada (Frederick, 2013). Desarrollar este tipo de entrevistas permite poder incidir y profundizar en aspectos relacionados con la temática, lo que favorece la obtención de datos más veraces y aplicables (Smith y Osborn, 2003).

Teniendo en cuenta el tipo de análisis realizado, y atendiendo a los dos factores de estudio, fueron cinco las cuestiones iniciales preguntadas a los dos profesores.

1. ¿Consideras importante que el alumno trabaje aspectos prácticos en la asignatura? ¿Cómo lo fomentas en el aula? 2. ¿Es fundamental que en la asignatura se trabajen recursos útiles que el alumno pueda utilizar fuera del aula? ¿De qué manera lo trabajas? 3. ¿Qué tipo de competencias docentes trabajas en clase? 4. ¿Crees que el alumno valora que en clase se trabajen aspectos relacionados con competencias profesionales de un futuro profesor? ¿Por qué? 5. ¿Qué metodología utilizas para que el alumno se implique en su aprendizaje? 
La totalidad de información recabada se ha agrupados en tres categorías para su posterior análisis: 1. "Practicidad en el tipo de actividades trabajadas", 2. "Desarrollo de capacidades docentes en la asignatura y 3. "Metodología utilizada para fomentar la transferencia de competencias profesionales".

\section{Diseño y procedimiento}

A pesar de que todos los alumnos hayan cursado la misma asignatura, ésta ha sido dividida en dos grupos, el A impartido por un profesor en horario de mañana y el B impartido por otro por la tarde. Consecuentemente y a pesar de que la guía docente es la misma para los dos grupos, y se trabajan las mismas competencias y objetivos, la metodología empleada es diferente. Cada uno presenta las siguientes características:

Grupo A: se ha empleado una evaluación formativa a lo largo de la asignatura, delimitando desde el comienzo los criterios de evaluación y de calificación de cada uno de los procedimientos empleados. Además ha existido un registro del trabajo semanal por cada uno de los grupos, lo que ha favorecido tanto su autoevaluación grupal como la coevaluación de cada uno de sus integrantes. Para ello, se han empleado las rúbricas y las escalas de valoración que utiliza el docente para calificar cada una de las actividades al finalizar el curso.

Grupo B: la metodología utilizada presenta unas características más tradicionales, ya que es el docente el que tiene el principal peso tanto en la evaluación como en el proceso de enseñanza y aprendizaje. No existe un registro del trabajo por grupos ni una retroalimentación a lo largo de la asignatura, lo que deriva en que no se empleen autoevaluaciones ni coevaluaciones entre los miembros de la clase. Las sesiones son más directivas, y el alumno actúa en función del contenido que se va impartiendo en cada sesión.

Como el diseño empleado es pretest-postest, los alumnos cumplimentan el cuestionario de manera individual antes del comienzo de la asignatura y una vez que ha finalizado la misma. Se garantizó tanto el anonimato como la confidencialidad en el tratamiento de los datos, buscando así la veracidad en las respuestas. Además se les insistió en que sus respuestas no tendrían ninguna influencia ni repercusión en la calificación de la asignatura.

\section{Análisis empleado}

La metodología de la investigación ha sido mixta, empleándose tanto un análisis cuantitativo (descriptivo e inferencial) como cualitativo (entrevistas). Se emplea un pretest-postest, comprobando en qué medida varía la percepción del alumnado sobre la adquisición de competencias profesionales docentes tras haber cursado la asignatura. Estos datos se complementan con la valoración de los docentes una vez finalizado el proceso. Esta complementariedad en el tratamiento de los datos otorgará un visionado más global de los resultados obtenidos, así como una mayor comprensión de los mismos. Además, favorece la transformación de los procesos educativos desarrollados (Hall y Ryan, 2011). 


\section{Cuantitativo}

Se ha utilizado un tratamiento descriptivo (medias y DT) y uno inferencial (correlaciones, y Anovas) para la totalidad de los alumnos que han cursado la asignatura. Dentro del análisis descriptivo se utiliza un diseño de medidas repetidas (DMR) de carácter longitudinal antes y después, utilizando un modelo condicional.

Este análisis, mediante el paquete estadístico SPSS, se realiza para obtener la percepción del alumnado sobre la transferencia del aprendizaje obtenido a futuras competencias profesionales docentes, comprobando si varía significativamente en función de la metodología utilizada en cada uno de los grupos.

\section{Cualitativo}

Este tipo de análisis que complementa al cuantitativo se ha obtenido a partir de la realización de las entrevistas comentadas. Tal y como establecen Libarkin y Kurdziel (2002) se centra en el análisis profundo del contenido estudiado, contrastando los patrones de texto literales extraídos de las entrevistas y vinculándolos a cada una de las categorías generadas. Para ello, se triangula y se satura la información recabada de cada variable analizada, codificando los extractos coincidentes con los patrones cruzados (Saldaña, 2009). Todos los investigadores, un total de tres, revisaron y consensuaron de manera crítica y reflexiva los primeros datos tras su volcado. La confiabilidad fue apoyada a través de la retroalimentación continua y el análisis participativo por parte de los investigadores que revisaron y perfeccionaron las categorías emergentes, para que los resultados pudieran ser considerados confiables, creíbles y transferibles (Cooper, 2014). Se utilizan los extractos de textos literales obtenidos en las respuestas para reflejar la veracidad de las percepciones, reflejando los más significativos de cada categoría (Cohn, 1991). Este aporte de información cualitativa favorece la profundización y la comprensión de los resultados desde una perspectiva más amplia.

Para recapitular, organizar y obtener la saturación de los datos a partir de las categorías generadas en las preguntas realizadas a los dos docentes se ha utilizado el programa de computación WEFT QDA, que favorece el registro, la catalogación y la asignación de criterios comunes a la información obtenida. Se utiliza una codificación para cada uno de los docentes, con el fin de vincular cada extracto de texto mostrado. Para el profesor del grupo A se utiliza el código EPGA (entrevista profesor grupo A) y para el B EPGB (entrevista profesor grupo B).

\section{Resultados}

\section{Análisis descriptivos. Contraste de percepción de alumnado pre-post entre grupos}

Tal como se puede observar en la tabla 1, en el pretest no existen diferencias significativas en los factores entre ninguno de los dos grupos, obteniendo valores medios más elevados en el grupo B. Sin embargo, en el postest, y dentro del grupo A, se obtienen diferencias significativas respecto al pretest, observando cómo los alumnos en este grupo han experimentado un incremento sustancial en relación a la adquisición de las 
competencias docentes. En este factor también se alcanzan diferencias entre grupos. El grupo A en el factor uno obtiene también una media más elevada que en el grupo B.

Tabla 1. Comparación de medias por factores para cada uno de los grupos en el pretest-postest (nivel de significación en las diferencias: $p<.05$ )

\begin{tabular}{lccccccc}
\hline & \multicolumn{3}{c}{ PRE-TEST } & \multicolumn{3}{c}{ POST-TEST } \\
\cline { 2 - 9 } & N & Media & DT & Var. & Media & D.T & Var. \\
\cline { 2 - 8 } & \multicolumn{5}{c}{ Grupo A } \\
\hline F.1. Aplicabilidad aprendizaje & 63 & 3.24 & .205 & .042 & 4.03 & .102 & .010 \\
\hline F.2. Competencias docentes & 63 & 3.51 & .135 & .018 & $4.62^{\text {aa* }}$ & .093 & .086 \\
\hline & \multicolumn{5}{c}{ Grupo B } \\
\hline F.1. Aplicabilidad aprendizaje & 50 & 3.48 & .318 & .101 & 3.53 & .134 & .017 \\
\hline F.2. Competencias docentes & 50 & 3.86 & .250 & .062 & $3.65^{\text {ba* }}$ & .219 & .047 \\
\hline
\end{tabular}

*Diferencias entre el pretest y postest en el grupo A en el factor 2

**Diferencias en el postest entre el grupo B y A en el factor 2

\section{Análisis inferencial: ANOVA. Análisis de la utilidad de la experiencia}

Se ha realizado un análisis Bonferroni y Post Hoc. A partir del análisis factorial realizado y en relación a los ítems relacionados con aprovechamiento del aprendizaje y su extrapolación a otros ámbitos, se ha creado la variable de escala denominada "utilidad del aprendizaje adquirido". Se busca la interacción entre esta variable dependiente y otras independientes. La primer de ellas es la edad; 1. "entre 20 y 22 ", 2. "entre 22 y 25 ". 3. "más de 25". La segunda es las titulaciones universitarias previas, categorizada en, 1. "ninguna", 2. "una titulación", 3. "más de una titulación". La última se refiere a la experiencia en autoevaluaciones y coevaluaciones, 1. "ninguna experiencia", 2. "entre una y dos experiencias", 3. "más de tres experiencias" (ver tabla 2).

Tabla 2. Resumen de Anova (Bonferroni) para cada una de las variables independientes analizadas en el postest (edad, titulaciones previas y experiencia en autoevaluaciones y coevaluaciones)

\begin{tabular}{|c|c|c|c|}
\hline UTILIDAD DEL APRENDIZAJE ADQUIRIDO & $F$ & $g l$ & $p$ \\
\hline \multicolumn{4}{|l|}{ Grupo A } \\
\hline Edad & 103.21 & 1 & .316 \\
\hline Titulaciones universitarias previas & 92.51 & 2 & $.015^{*}$ \\
\hline Experiencia autoevaluaciones y coevaluaciones & 66.21 & 1 & .151 \\
\hline \multicolumn{4}{|l|}{ Grupo B } \\
\hline Edad & 95.31 & 1 & .184 \\
\hline Titulaciones previas & 87.45 & 1 & .231 \\
\hline Experiencia autoevaluaciones y coevaluaciones & 91.42 & 2 & $.022^{* *}$ \\
\hline
\end{tabular}


Se observa cómo en el grupo A las diferencias se obtienen en relación a la variable de titulaciones previas $\left(\mathrm{F}_{(60)}=92,51, \mathrm{p}<.015\right)$, siendo los que tienen más de una titulación los que valoran más positivamente el aprendizaje adquirido. Sin embargo, en el grupo B las diferencias se encuentran en la variable independiente de las experiencias previas en autoevaluaciones y coevaluaciones $\left(F_{(53)}=91,42, p<.022\right)$. En concreto, los alumnos de este grupo que han tenido mayores experiencias a lo largo de la carrera relacionadas con estas estrategias evaluativas valoran menos la utilidad del aprendizaje adquirido en esta asignatura.

\section{Análisis cualitativo de la percepción del docente}

De toda la información extraída en las respuestas de las entrevistas realizadas a los profesores participantes, la información se presenta en función de las 3 categorías generadas: 1- "Practicidad en el tipo de actividades trabajadas", 2- "Desarrollo de capacidades docentes en la asignatura" y 3- "Metodología utilizada para fomentar la transferencia de competencias profesionales".

\section{Practicidad en el tipo de actividades trabajadas}

Se observa cómo el profesor que llevó a cabo las autoevaluaciones y coevaluaciones a lo largo de la asignatura entiende que la practicidad ha de estar vinculada a un "para qué" de la enseñanza, asociado a una autonomía por parte del alumno. Sin embargo, el profesor del grupo B únicamente aboga por la práctica sin asociarla a unos fines de aprendizaje concretos, delegando menos en los alumnos.

Es fundamental que el alumno sea protagonista de su propio aprendizaje, pero para ello es necesario vincular lo que hacemos en el aula con unos fines de aprendizaje claros, dejando al alumno que demuestre lo que ha aprendido [...] (EPGA)

Entiendo la práctica como la vivencia de aquello que se explica en clase [...] (EPGB)

Prefiero ser yo el que proponga, así me aseguro verdaderamente de lo que el alumno está haciendo (EPGB)

\section{Desarrollo de capacidades docentes en la asignatura}

El docente del grupo A vincula más las tareas que propone en el aula al desarrollo de capacidades propias de un docente, argumentando que en la titulación se está formando a futuros maestros. El del grupo B no dota a la asignatura de esa finalidad más transversal, ya que se centra en la adquisición propia de los objetivos marcados en la guía docente.

Bastante tenemos con que los alumnos aprendan lo que enseñamos en las asignaturas como para pretender otras cosas [...] (EPGB)

Si cada profesor enseñara lo que debe mucho cambiaría el panorama [...] (EPGB)

Creo que en esta titulación deberíamos trabajar más conjuntamente de lo que los hacemos $[\ldots]$ (EPGA) 
En todas las asignaturas hay aspectos comunes que deberá adquirir un futuro docente, y es así donde debemos de poner el punto de mira (EPGA)

\section{Metodología utilizada para fomentar la transferencia de competencias profesionales}

En consonancia a la categoría anterior, el profesor que ha llevado a cabo las autoevaluaciones y coevaluaciones con los alumnos otorga una mayor relevancia al rol que desempeña la metodología, sobre todo en la transferencia del aprendizaje.

Si queremos que el alumno transfiera fuera del aula lo que le enseñamos es prioritario utilizar metodologías abiertas y participativas que garanticen y lo posibiliten [...] (EPGA)

Nosotros enseñamos lo que nos corresponde [...] (EPGB)

Luego la profesión que decidan o que puedan desempeñar los alumnos es otra cosa, pero seguro que utilizan algo de lo que les hemos enseñado (EPGB)

\section{Discusıón}

Se ha observado en la investigación cómo la metodología llevada a cabo en el aula en cada uno de los grupos ha sido un factor determinante para modificar la percepción del alumnado sobre la adquisición de competencias docentes y su transferencia al futuro ámbito profesional. El grupo que vivenció las autoevaluaciones y coevaluaciones como elemento formativo valoró más el aprendizaje recibido. Además, dentro de este grupo fueron los alumnos que más titulaciones previas tienen los que percibieron una mayor utilidad del aprendizaje. Dentro del grupo que no utilizó la metodología abierta y participativa las diferencias se encontraron en la variable del número de veces que habían participado en su propio proceso de evaluación, siendo los que más veces lo han hecho los que menos utilidad encuentran en el aprendizaje han vivenciado.

En el pretest no se aprecian diferencias significativas entre grupos en ninguno de los dos factores del estudio, lo que permite comprobar fehacientemente los efectos que ha tenido el plan de intervención desarrollado, en este caso las metodologías aplicadas en la asignatura (Cahit, 2015). Las medias en el pretest en cada uno de los factores son más elevadas en el grupo B, lo que refleja que las experiencias positivas en las competencias docentes desarrolladas en otras asignaturas de ese curso o anteriores eran mayores. Es preciso destacar que el grupo A y B es el mismo para todas las asignaturas de las que consta la titulación, algo que favorece la veracidad de las percepciones generadas en el grupo. Lo que se pone de manifiesto es la variabilidad que presenta el alumnado en sus valoraciones, algo que indica la disparidad de criterios metodológicos de enseñanza que utilizan los docentes universitarios (Rodríguez y Álvarez, 2013).

Tras haber finalizado la asignatura, es el factor dos, relativo a la transferencia de las competencias docentes, en el que se obtienen las diferencias significativas en el grupo A, tanto en relación al pretest como en comparación con el grupo B. Esto refleja que la metodología empleada en la asignatura ha repercutido favorablemente en la percepción del alumno sobre sus capacidades como docente. Como indica 
García-Carmona (2013) si formamos futuros docentes hemos de constatar que estas competencias se adquieren en las asignaturas, estableciendo mecanismos metodológicos y evaluativos que garanticen el trabajo sobre contextos reales. Se refleja también que la diversa forma de abordar los contenidos de un grupo y otro en la asignatura repercute directamente en la percepción del alumno sobre el aprendizaje adquirido. Este hecho ha de tenerse muy en cuenta en el ámbito universitario, ya que es tan importante el qué se enseña como la manera en la que se hace, y más en asignaturas que trabajen temáticas similares (Hsieh, 2015). Por lo tanto, se observa cómo el alumno en la presente investigación ha interiorizado los procesos de autoevaluación grupal y de coevaluación intra e intergrupal como positivos para implicarse en su proceso de aprendizaje. Autores como Mulder, Baik, Naylor y Pearce (2014) indican que es fundamental que el alumno se someta y sea sometido a juicios de valor entre iguales, ya que esa retroalimentación es en muchos casos más efectiva que la heteroevaluación del profesor. Además, cuando a los grupos de trabajo se les permite que se autoevalúen mediante instrumentos consensuados y delimitados de antemano son más conscientes de lo que tienen que mejorar en el trabajo, obteniendo mejores resultados en el proceso final (Migueles y Hernández, 2014). Como consecuencia del resultado obtenido con la metodología puesta en práctica, en el factor uno, aunque sin llegar a diferencias significativas, el grupo A también obtiene una media más elevada que el B, lo que denota la mayor aplicabilidad que tienen los aprendizajes generados bajo la perspectiva del estudiante. Atender a las valoraciones de quien enseñamos solamente puede traer beneficios para la mejora, estableciéndose un ajuste entre lo que pretendemos enseñar y lo que verdaderamente se aprende (Snyder, 2010).

En cuanto a la influencia de las variables independientes sobre la percepción que tiene el alumno acerca de la utilidad del aprendizaje obtenido, en el grupo que llevó a cabo las autoevaluaciones y coevaluaciones las diferencias se encuentran en la variable de titulaciones previas, en concreto entre los que no tienen ninguna y los que tienen más de una. Son los que tienen más de una los que consideran más útiles la implantación de este tipo de metodologías abiertas y participativas. Por lo tanto, parece que los alumnos con más experiencia y trayectoria universitaria son más conscientes de los beneficios que conllevan estos modelos de aprendizaje, quizás porque no están muy acostumbrados a su experimentación. Experiencias como la de Boza y Méndez-Garrido (2013) indican que los alumnos con implicación hacia las tareas valoran muy positivamente métodos de trabajo en los que se les permita experimentar y tomar decisiones sobre la manera en la que regular su aprendizaje, siempre bajo el asesoramiento guiado del profesor. Por el contrario, en el presente estudio se presenta cómo los estudiantes que no tienen ninguna titulación universitaria valoran más positivamente el aprendizaje obtenido en el grupo B de la asignatura. Houghton y Ruth (2010) destacan que en primeros cursos los alumnos son menos críticos y reflexivos hacia los procesos de aprendizaje en los que se encuentran inmersos, debido a que no tienen el bagaje suficiente para poder valorar y contrastar con otros procedimientos evaluativos. En la variable de la edad no se han obtenido diferencias significativas en ninguno de los grupos. En este sentido, algunos estudios que utilizan la edad como variable independiente (Elsaadani, 2013) reflejan que los alumnos de más edad son 
más reacios y presentan más dificultades a la hora de trabajar en grupo, utilizar las nuevas tecnologías o asimilar procedimientos vinculados a metodologías de carácter innovador.

En relación a la parte cualitativa del estudio, la disparidad de criterios entre los docentes es manifiesta, tanto en relación al tipo de metodología empleada como en lo referente a los principales fines que tiene la docencia para futuros maestros. El docente del grupo A enfoca la asignatura hacia la adquisición de competencias transversales por parte del alumno, yendo un poco más allá que la enseñanza de los contenidos específicos de la materia y fomentando su autonomía en el aprendizaje. Sin embargo, el profesor del grupo B incide en que lo principal son los aprendizajes específicos de la asignatura, no contemplando vías evaluativas que garanticen la enseñanza de contenidos comunes. Cox (2015) refleja que en la actualidad todo docente universitario que forme a futuros docentes ha de reflexionar sobre la manera en la que la forma de trabajar en el aula está o no en conexión con las demandas que tendrá un futuro profesor, siempre garantizado la reproducción de situaciones de trabajo en contextos reales. En este sentido, se observa cómo el profesor que empleó las autoevaluaciones y coevaluaciones en el aula otorga más importancia a aspectos relacionados con la búsqueda de información, emisión de feedbacks, capacidad para sintetizar la información, análisis de material de aplicación al aula y diseño de sesiones, mientras que el docente del B estructura su asignatura a través del desarrollo de sesiones prácticas basadas en el juego aplicado a la EF. En consonancia con esto, y en relación a la tercera categoría de análisis, el profesor centra el proceso de aprendizaje en "enseñar a enseñar". Por el contrario, la profesora del grupo B lleva el peso fundamental en la impartición de contenidos, no favoreciendo la evaluación formativa enfocada a la mejora del trabajo a lo largo del proceso. Sin embargo, estrategias basadas en este tipo de evaluación demuestran una mayor implicación del alumno en el proceso, garantizando además un aprendizaje más significativo Wylie y Lyon (2015).

\section{Conclusiones}

En relación al primer objetivo de la investigación, se ha observado que en el grupo que se aplicó la metodología abierta y participativa fundamentada en el desarrollo de autoevaluaciones y coevaluaciones se obtuvieron unos valores más elevados tanto en la aplicabilidad de los aprendizajes como en la adquisición y transferencia de competencias docentes. Esto se relaciona con otras experiencias como las de Tylor (2014), que determinan que el empleo de las autoevaluaciones a lo largo del proceso favorecen el registro del trabajo y una mayor conciencia sobre lo que se está realizando.

Respecto al segundo objetivo, dentro del grupo A se obtuvieron diferencias en la variable de titulaciones previas, siendo los alumnos que más tenían los que más utilidad le dieron al aprendizaje obtenido. Sin embargo, en el grupo B las diferencias se encontraron en la variable de las experiencias previas en auto y coevaluaciones. Los que más experiencia tenían peor valoraron la metodología recibida. En este sentido, Sandvoll, (2014) indica que cuando un alumno ha vivenciado procesos de evaluación formativa que le han conducido a un mayor aprendizaje, se muestra reacio ante otros sistemas evaluadores que no lo permiten ser miembro activo del proceso. 
El tercer objetivo, referente a la parte cualitativa del estudio, refleja una disparidad de criterios de los dos profesores tanto en relación a la metodología desarrollada como a los fines de aprendizaje fundamentales que ha de tener un futuro maestro, siendo el docente del grupo A el que aboga por la inclusión de procedimientos que permitan al alumno ser el que regule su propio proceso de enseñanza. Otros estudios en esta línea reflejan la dificultad existente en ocasiones para que los profesores se pongan de acuerdo sobre principios comunes de actuación (Kise, 2014).

Consideramos que este artículo puede ser de interés para todas las autoridades encargadas de regular el sistema académico en el ámbito universitario. También para los docentes que quieran reflexionar sobre la importancia y relevancia que tienen las tareas propuestas en el aula en la percepción del alumnado sobre la transferencia del aprendizaje generado. El artículo presenta algunas limitaciones. En primer lugar, únicamente se atiende a la percepción del alumno en una asignatura, por lo que sería interesante contrastar estas valoraciones con otros cursos e incluso diferentes titulaciones. En segundo lugar, no se incide en covariables referentes a las características del profesorado, por lo que en futuras investigaciones podría profundizarse en cómo influye la formación inicial y permanente del profesorado en la manera de enseñar en el aula. No obstante, se plantea como futura línea de investigación la determinación de prácticas educativas de éxito que, independientemente de las titulaciones en las que se enmarquen, reflejen procedimientos claros de actuación para fomentar una transferibilidad de los aprendizajes.

\section{ReFERENCIAS BIBLIOGRÁFICAS}

Baena, A., Granero, A., Sánchez, J. A., y Martínez, M. (2013). Apoyo a la autonomía en Educación Física: antecedentes, diseño, metodología y análisis de la relación con la motivación en estudiantes adolescentes. Retos: nuevas tendencias en educación física, deporte y recreación, 24, 46-49.

Boyle, T., y Petriwskyj, A. (2014). Transitions to School: Reframing Professional Relationships. Early Years: An International Journal of Research and Development, 34(4), 392-404.

Boza, A., y Méndez-Garrido, J. M. (2013). Aprendizaje motivado en alumnos universitarios. Validación y resultados generales de una escala. Revista de investigación educativa, RIE, 31(2), 331-347.

Cahit, K. (2015). Internal Validity: A Must in Research Designs. Educational Research and Reviews, 10(2), 111-118.

Castejón, F. J., Santos, M., y Palacios, A. (2013). Cuestionario sobre metodología y evaluación en formación inicial en Educación Física. Revista Internacional de Medicina y Ciencias de la Actividad Física y el Deporte, 12(1), 1-23.

Chang, C., Liang, C., y Chen, Y. (2013). Is Learner Self-Assessment Reliable and Valid in a Web-Based Portfolio Environment for High School Students? Computers y Education, 60(1), 325-334.

Cohn, P. J. (1991). An exploratory study on peak performance in golf. The Sport Psychologist, 5, 1-14. 
Cooper, K. (2014). Eliciting Engagement in the High School Classroom: A MixedMethods Examination of Teaching Practices. American Educational Research Journal, 51(2), 363-402.

Corbetta, P. (2007). Metodologías y técnicas de investigación social. Madrid: McGrawHill.

Cox, S. (2015). The Future of Primary Education. FORUM: for promoting 3-19 comprehensive education, 57(1), 27-30.

Elsaadani, M. A. (2013). Exploring the Relationship between Teaching Staff Age and Their Attitude towards Information and Communications Technologies (ICT). Online Submission, International Journal of Instruction, 6(1), 216-226.

Frederick, A. (2013). The Influence of Power Shifts in Data Collection and Analysis Stages: A Focus on Qualitative Research Interview. Qualitative Report, 18, 15-21.

García-Carmona, A. (2013). Educación científica y competencias docentes. Análisis de las reflexiones de futuros profesores de Física y Química. Revista Eureka sobre enseñanza y divulgación de las ciencias, 10(4), 552-567.

González-Garzón, M.L., y Laorden, C. (2012). El Prácticum en la formación inicial de los maestros en las nuevas titulaciones de Educación infantil y primaria. El punto de vista de profesores y estudiantes. Pulso: revista de educación, 35, 131-154.

Hortigüela, D., Pérez Pueyo, A., y Abella, V. (2015a). Perspectiva del alumnado sobre la evaluación tradicional y la evaluación formativa. Contraste de grupos en las mismas asignaturas. REICE. Revista Iberoamericana sobre Calidad, Eficacia y Cambio en Educación, 13(1), 35-48. Recuperado de http://www.rinace.net/reice/ numeros/arts/vol13num1/art3.pdf.

Hortigüela, D., Pérez-Pueyo, A., y Abella, V. (2015b). ¿Cómo influye el sistema de evaluación en la percepción del alumnado? @TIC revista d'innovació educative, 14, 82-89. doi: 10.7203/attic.14.4170

Houghton, L., y Ruth, A. (2010). Making Information Systems Less Scrugged: Reflecting on the Processes of Change in Teaching and Learning. Journal of Information Technology Education, 9, 91-102.

Hsieh, B. (2015). The Importance of Orientation: Implications of Professional Identity on Classroom Practice and for Professional Learning. Teachers and Teaching: Theory and Practice, 21(2), 178-190.

Janssen, F., Westbroek, H., y Doyle, W. (2015). Practicality Studies: How to Move from What Works in Principle to What Works in Practice. Journal of the Learning Sciences, 24(1), 176-186.

Jiménez, A. (2009). Reflexiones sobre la necesidad de acercamiento entre universidad y mercado laboral. Revista Iberoamericana de Educación, 50(1), 1-9.

Kise, J. (2014). Two Sides of the Coin: Evaluation and Support Work Together to Strengthen Teaching. Journal of Staff Development, 35(6), 24-28.

Lappalainen, J., y Rosqvist, J. (2015). Exploring Hurdles to Transfer: Student Experiences of Applying Knowledge across Disciplines. International Journal of Mathematical Education in Science and Technology, 46(3), 404-419.

Libarkin, J.C., y Kurdziel. J.P. (2002). Research methodologies in science education: Qualitative data. Journal of Geoscience Education, 50, 195-200. 
MacDonald, B. (2012). Using Self-Assessment to Support Individualized Learning. Mathematics Teaching, 231, 26-27.

Maritz, J., y Jooste, K. (2011). Debriefing Interviews and Coaching Conversations: Strategies to Promote Student Reflexivity and Action. South African Journal of Higher Education, 25(5), 972-986.

Migueles, A., y Hernández, A. (2014). Desmitificando algunos sesgos de la autoevaluación y coevaluación en los aprendizajes del alumnado. REXE: Revista de estudios y experiencias en educación, 13(25), 13-31.

Mulder, R., Baik, C., Naylor, R., y Pearce, J. (2014). How Does Student Peer Review Influence Perceptions, Engagement and Academic Outcomes? A Case Study. Assessment y Evaluation in Higher Education, 39(6), 657-677.

Ozcan, K. (2013). Student Evaluation of Lecture and Teaching Effectiveness in Higher Education. Educational Research and Reviews, 8(8), 378-389.

Rodríguez, A., y Álvarez, E. (2013). Metodologías y recursos en las titulaciones de Grado: Perspectiva de estudiantes y responsables institucionales. Revista electrónica interuniversitaria de formación del profesorado, 16(2), 105-120.

Rodríguez-Sánchez, M. (2011). Metodologías docentes en el EEES: de la clase magistral al portafolio. Tendencias pedagógicas, 17, 83-103.

Romero, L., Orzechowski., A., y Rahatka, O. (2014). Teaching Problem-Solving and Critical-Thinking Skills Online Using Problem-Based Learning. Educational Technology, 54(1), 20-25.

Saldaña, J. (2009). The Coding Manual for Qualitative Researchers. Thousand Oaks, CA: Sage.

Sandvoll, R. (2014). Feeling Good, but Missing the Mark. What Happened to the Assessment in Peer Assessment? Journal of University Teaching and Learning Practice, 11(1), 11-21.

Smith, J. A., y Osborne, M. (2003). Interpretative Phenomenological Analysis. En J.A. Smith (Ed.) Qualitative psychology: A practical guide to research methods. (pp. 53-79). London: Sage.

Snyder, L. (2010). The Use of Pre-Group Instruction to Improve Student Collaboration. Journal of Applied Research for Business Instruction, 8(1), 1-12.

Teijeiro, M., Rungo, P., y Freire, M.J. (2013). Graduate Competencies and Employability: The Impact of Matching Firms' Needs and Personal Attainments. Economics of EducationReview, 34, 286-295.

Torrego, L., y Ruíz, C. (2011). La coordinación docente en la implantación de los títulos de Grado. Revista electrónica interuniversitaria de formación del profesorado, 14(4), 31-40.

Trainor, A., y Graue, E. (2014). Evaluating Rigor in Qualitative Methodology and Research Dissemination. Remedial and Special Education, 35(5), 267-274.

Trede, F., y McEwen, C. (2015). Early Workplace Learning Experiences: What Are the Pedagogical Possibilities beyond Retention and Employability? Higher Education: The International Journal of Higher Education and Educational Planning, 69(1), 19-32. 
Trigueros, C., Rivera, E., y De la Torre, E. (2012). La evaluación en el aula universitaria: del examen tradicional a la autoevaluación. Revista Internacional de Medicina y Ciencias de la Actividad Física y del Deporte, 47, 1-6.

Tylor, S. (2014). Student Self-Assessment and Multisource Feedback Assessment: Exploring Benefits, Limitations, and Remedies. Journal of Management Education, 38(3), 359-383.

Van Loon, A. M., Ros, A., y Martens, R. (2013). Designing Digital Problem Based Learning Tasks that Motivate Students. Journal of Technology and Teacher Education, 21(4), 409-432.

Wylie, E. C., y Lyon, C. J. (2015). The Fidelity of Formative Assessment Implementation: Issues of Breadth and Quality. Assessment in Education: Principles, Policy y Practice, 22(1), 140-160. 